\title{
Publisher Correction: Human-chimpanzee fused cells reveal cis-regulatory divergence underlying skeletal evolution
}

David Gokhman (1), Rachel M. Agoglia, Maia Kinnebrew (1D, Wei Gordon (1D, Danqiong Sun, Vivek K. Bajpai (D), Sahin Naqvi (1), Coral Chen (1), Anthony Chan (1), Chider Chen, Dmitri A. Petrov, Nadav Ahituv (1), Honghao Zhang, Yuji Mishina (1), Joanna Wysocka (1), Rajat Rohatgi (1) and Hunter B. Fraser(1)

Correction to: Nature Genetics https://doi.org/10.1038/s41588-021-00804-3, published online 17 March 2021.

In the version of this article initially published, in Fig. 1b, the graphs contained stray vertical lines, owing to a figure-processing error. The error has been corrected in the HTML and PDF versions of the article.
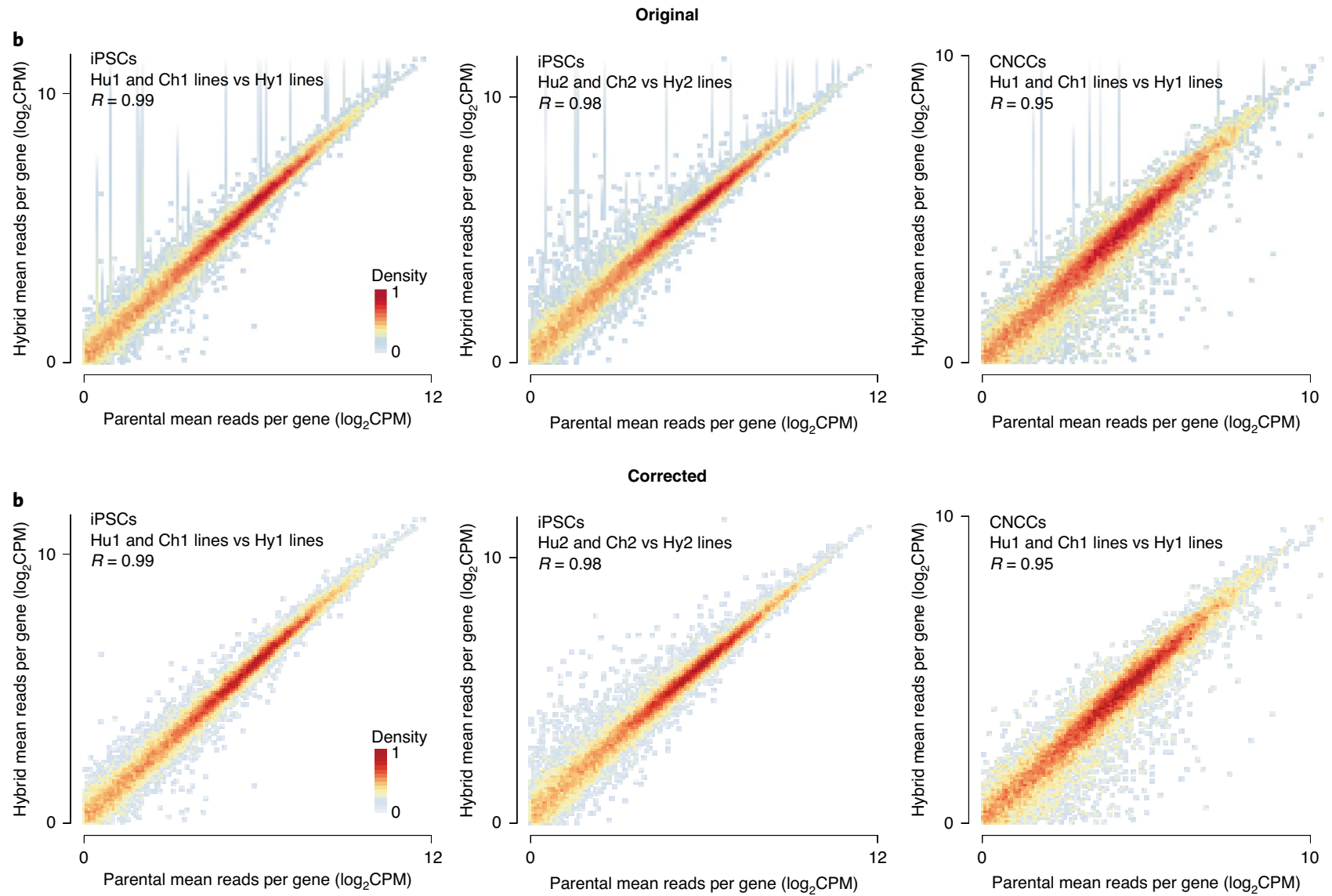

Fig. 1 | Original and corrected.

Published online: 24 March 2021

https://doi.org/10.1038/s41588-021-00849-4

(๑) The Author(s), under exclusive licence to Springer Nature America, Inc. 2021 